\title{
Aumento de la conducta operante tras la presentación de estímulos condicionados asociados al efecto del etanol
}

\author{
Increments in operant behavior due to a CS associated to \\ ethanol
}

Vanetza Quezada Scholz, Daniel Alarcón Acuña, Gonzalo Miguez Cavieres, Ronald Betancourt Mainhard

\begin{abstract}
Resumen
Estudios previos señalan que estímulos externos asociados a drogas adquieren propiedades motivacionales que llegan a controlar la respuesta operante de búsqueda/consumo de droga y otro tipo de recompensas. La presente investigación tiene como objetivo demostrar que los ECs asociados al efecto del etanol adquieren propiedades motivacionales modificando la tasa de respuesta operante para la obtención de comida. Para ello se realizó un experimento de transferencia de control con ratas albinas. Al grupo experimental se le hicieron presentaciones contingentes del EC (tono) y el estímulo incondicionado (EI) (i.e., efecto del etanol). Los sujetos del grupo control tuvieron exposiciones aleatorias EC/EI. Posteriormente se comparó la tasa de respuesta operante para la búsqueda de comida frente al EC al etanol en el grupo experimental y control. Los resultados indican que los ECs asociados al efecto del etanol aumentan la respuesta operante frente a reforzadores naturales.

Palabras clave: condicionamiento clásico, condicionamiento operante, transferencia de control, tolerancia a las drogas.
\end{abstract}

1 Vanetza Quezada Scholz. Universidad de Chile, Santiago, Chile. qvanetza@u.uchile.cl Daniel Alarcón Acuña. Universidad de Chile, Santiago, Chile. dealarcon@gmail.com

Gonzalo Miguez Cavieres. State University of New York at Binghamton, Nueva York, Estados Unidos.gmiguez1@binghamton.edu

Ronald Betancourt Mainhard. Universidad de Chile, Santiago, Chile. rbetancourt@progranja.cl 


\begin{abstract}
Previous studies have shown that stimuli associated with drugs can acquire motivational proprieties, which allow them to control operant behavior for drug consumption/seeking and other types of reward. The current research investigated whether a conditioned stimulus (CS) that has been paired with ethanol is able to disrupt the rate of responding for a reward. A Pavlovian instrumental transfer experiment was conducted with albino rats. The experimental group received paired presentations of the CS (i.e, tone) and the unconditioned stimulus (US) (i.e., the effects of ethanol). Subjects in the control group received random presentations of the US and the CS. The rate of operant behavior for food seeking in both the presence and the absence of the CS was determined for the subjects. The results show that a CS associated with ethanol increased the rate of responding for food.
\end{abstract}

Key words: Pavlovian conditioning, Operant conditioning, Pavlovian instrumental transfer, Drug tolerance.

\title{
Introducción
}

En el abordaje psicológico de las adicciones, uno de los campos más destacables es la investigación básica abocada a la búsqueda de los mecanismos subyacentes que podrían explicar la conducta adictiva, y es en esta línea donde el estudio de los procesos de aprendizaje asociativo ha realizado un gran aporte tanto desde las investigaciones enmarcadas en el condicionamiento pavloviano (Betancourt 2006; Betancourt, Inostroza y Laborda, 2008; Betancourt, Díaz y Quezada, 2009; Betancourt, Corada, Dominichetti, Laborda, Martínez y Miguez, 2008; Larson \& Siegel, 1998; McDonald \& Siegel, 2004; Siegel \& Ramos, 2002) como en el condicionamiento operante (Ahmed \& Koob, 1997; Di Ciano \& Everitt, 2003; Hellemans, Dickinson \& Everitt, 2006; Miles, Everitt, Dalley \& Dickinson, 2004; Shalev, Highfield, Yap \& Shaham, 2000; Shaham \& Miczek, 2003).

Desde el condicionamiento pavloviano, la conducta adictiva ha sido explicada a través del modelo de respuestas compensatorias (Crowell, 
Hinson \& Siegel, 1981; Goodinson \& Siegel, 1995; MacRae \& Siegel, 1997; Siegel, 1977, 1978, 1991, 1999, 2001; Siegel \& Larson, 1996), el que plantea que las claves asociadas (estímulos condicionados [ECs]) con el consumo de droga, adquieren la capacidad de elicitar respuestas condicionadas (RCs) que se oponen al efecto perturbador de la droga en el organismo (estímulo incondicionado [EI]) preparándolo para su consumo (Betancourt, 2006; Ramos, Siegel \& Bueno, 2002). De este modo se resguarda la homeostasis fisiológica del organismo y se produce lo que se conoce como tolerancia a la droga, es decir, la disminución del efecto de una misma cantidad de droga tras repetidas administraciones (Betancourt, Díaz y Quezada, 2008).

Por otra parte, desde el condicionamiento operante, las investigaciones se basan en el estudio de los mecanismos de recompensa sobre la conducta de búsqueda de droga (Mayorga, Albañil y Cómbita, 2006). De esta manera se utilizan ensayos de auto-administración de drogas, en los cuales los sujetos, por medio de operantes libres, logran la dosis de droga determinada por el experimentador, las que son vistas como refuerzos (Dickinson, Wood \& Smith, 2002).

Del mismo modo se han realizado investigaciones que intentan dilucidar el tipo de interacción que se da entre los estímulos asociados pavlovianamente al efecto de la droga y las conductas operantes de búsqueda y consumo de droga. Al respecto se ha observado que los ECs asociados con drogas pueden influenciar la persistencia de la conducta adictiva induciendo el craving y la recaída en humanos adictos (Childress, Mozley, McElgin, Fitzgerald, Reivich \& O’Brien, 1999), así como también manteniendo la búsqueda de droga incluso después de prolongados periodos libres de droga en humanos y animales (Gawin y Kleber, 1986; Robinson y Berridge, 1993). Asimismo, Parkinson, Olmstead, Burns, Robbins y Everitt (1999) plantean que los estímulos asociados al consumo de droga pueden actuar como reforzadores condicionados, aumentando las conductas operantes de búsqueda y consumo.

Si bien existen numerosas investigaciones en el área, aún no existe consenso sobre el mecanismo a través del cual los estímulos asociados a los 
efectos de la droga logran controlar las respuestas operantes de búsqueda y consumo. Al respecto, investigaciones en las que se ha observado que las ratas evitan claves y contextos previamente pareados con la abstinencia a los opiáceos (Mucha, Gritti y Kim, 1986) se centran en los mecanismos de reforzamiento negativo para explicar el efecto que tienen los ECs asociados a la droga sobre la respuesta operante. Desde este punto de vista, las claves y contextos (ECs) asociados al consumo elicitan un estado motivacional aversivo frente al cual la conducta de consumo de droga le permite al organismo escapar o evitar las sensaciones desagradables originadas por el no consumo o abstinencia una vez que se ha establecido la dependencia (Bechara, Nader \& Van Der Kooy, 1998).

Por otro lado, se ha observado que cuando las ratas han tenido una experiencia previa con el consumo de heroína en presencia de un EC, la búsqueda de droga se eleva en presencia de este estímulo (Hellemans, Dickinson \& Everitt, 2006). Lo anterior no puede ser explicado por reforzamiento negativo, pues la búsqueda de heroína no termina en el consumo de ésta. Más bien la búsqueda de droga es una conducta dirigida a meta, ya que la experiencia de ingesta de droga bajo abstinencia aumenta su valor de incentivo.

Con el objetivo de comprender el rol que juegan los estímulos condicionados a la droga sobre las respuestas operantes, se realizan diseños de transferencia de control, cuyo propósito es observar los cambios producidos en la conducta de búsqueda de droga tras las manipulaciones de los ECs (Di Ciano \& Everitt, 2003), encontrándose evidencia de una influencia motivacional por parte estos estímulos.

Los diseños de transferencia de control son implementados mediante entrenamiento en tres fases. En la primera de ellas, denominada fase operante, se entrena una conducta específica, por ejemplo apretar palanca, por medio de la entrega de un reforzador. En la segunda fase pavloviana se asocia un EC al reforzador previamente entrenado (EI). Por último, en la fase de transferencia de control, se expone al sujeto a la presentación del EC durante la ejecución de la conducta operante y se miden los cambios en la ejecución producto del agregado del EC (Glasner, Overmier \& Balleine, 2005). 
En la actualidad existe evidencia contradictoria respecto del efecto que tienen los estímulos condicionados al etanol, específicamente a través de experimentos de transferencia de control. Al respecto, Krank (2003) encontró que la presentación de un EC, pareado previamente de manera contingente con la entrega de etanol, aumenta la conducta operante de consumo de etanol; en cambio un EC pareado de manera no contingente al reforzador no tiene efecto alguno sobre la conducta. Siguiendo la misma línea, Corbit y Janak (2007) realizaron experimentos cuyos datos replican los hallazgos de Krank, además de encontrar evidencia que indica la ocurrencia del mismo fenómeno cuando se utilizan refuerzos naturales (por ejemplo sucrosa). Lo interesante de este último experimento es que en el caso de los refuerzos naturales existiría cierta especificidad al momento de ejercer la influencia sobre la respuesta operante. Así, un EC asociado durante la fase pavloviana con la entrega de sucrosa sólo afecta la respuesta operante asociada previamente con la entrega de sucrosa como refuerzo, no afectando aquellas conductas que han sido reforzadas por otros estímulos. Por el contrario, un EC asociado con etanol afecta de manera generalizada la conducta operante asociada con cualquier reforzador, sea etanol o no.

Por otro lado, y contrario a los resultados de Krank (2003) y de Corbit y Janak (2007), Glasner et al. (2005) encontraron que las claves asociadas a la entrega de refuerzo, tanto etanol como un refuerzo natural, afectan de manera generalizada la conducta operante. Ellos no encontraron diferencias al comparar sujetos dependientes al etanol con sujetos no dependientes. Es decir, los ECs asociados en la fase pavloviana a la entrega del refuerzo natural aumentaron la conducta operante tanto para el mismo refuerzo como para el etanol, en ambos grupos.

Frente a lo anterior, el objetivo de la presente investigación es demostrar cómo los ECs asociados al efecto del etanol en ratas afectan la tasa de una respuesta operante mantenida con comida. Los resultados fueron consistentes con los supuestos de la Moderna Teoría de los dos Procesos (Rescorla \& Solomon, 1967), los cuales sostienen que el condicionamiento clásico provee las bases motivacionales para la conducta operante, esto se demuestra por el efecto de los estímulos condicionados 
sumados a la ejecución de ésta. Para tales efectos se realizó un experimento de transferencia de control. En la fase de condicionamiento clásico se entrenó a las ratas del grupo experimental para que asociaran un EC (Tono) con el efecto de una dosis de etanol de $2 \mathrm{gr} / \mathrm{kl}$ de peso (EI). Los sujetos del grupo control tuvieron exposiciones aleatorias EC/EI. Posteriormente se comparó la tasa de respuesta operante para la obtención de comida frente al EC asociado al etanol y en ausencia de éste.

\section{Método}

\section{Sujetos}

Se utilizaron 18 ratas albinas machos (Sprague-Dawley), de tres meses de vida y con un peso promedio de 220 gramos, las que fueron divididas aleatoriamente en dos grupos experimental y control. Las ratas se mantuvieron en jaulas individuales con alimento y agua ad libitum durante la fase pavloviana, mientras que durante la fase operante y el test de transferencia se mantuvieron bajo el protocolo de deprivación de Michael (1963).

\section{Aparatos}

Durante la Fase Pavloviana se utilizó un plano de deslizamiento (tilting plane), construido en el laboratorio de la Universidad de Chile, según un diseño estandarizado para medir ataxia en los procedimientos en que se utiliza etanol (Siegel \& Larson, 1996; Larson \& Siegel, 1998). Éste es un corredor de $60 \mathrm{cms}$. de largo por $10 \mathrm{cms}$. de ancho, rodeado de cuatro paredes de $30 \mathrm{cms}$. de alto. Tanto las paredes como el piso son de acrílico transparente. Un extremo de la caja está fijado con bisagras, en el cual se encuentra un transportador que mide los grados de inclinación de la caja. El otro extremo está conectado a una piola, la cual es accionada por una manivela. Un giro completo de ésta eleva el plano en 8 grados, con una velocidad de 4 grados por segundo. La ataxia se midió según el grado en que la rata comienza a deslizarse en el plano de inclinación. 
En la Fase operante y el Test de Transferencia se utilizaron cuatro cajas operantes (30 cms. de largo x $24 \mathrm{cms}$. de ancho x $20 \mathrm{cms}$. de alto). Cada caja cuenta con una palanca removible que se presiona hacia abajo. Además posee un dispensador que entrega $45 \mathrm{mg}$. de pellets Noyes (fórmula F) sobre un comedero ubicado bajo éste. El dispensador fue controlado a través del sistema ABET (Animal Behavior Environment Test) de Lafayette-Instrument (C).

\section{Estímulos}

Se utilizó como EI el efecto de una dosis de etanol al 95\% en una solución al $20 \%$ en suero fisiológico inyectada intraperitonealmente. La dosis por sujeto fue de 2 gramos de etanol por kilogramo de peso. Cuando no correspondía entregar el EI se utilizó una inyección intraperitoneal de suero fisiológico equivalente al volumen de la dosis de etanol (esta dosis es similar a la utilizada por Betancourt, Corada, Dominichetti, Laborda, Martínez y Miguez, 2008; Betancourt, Díaz y Quezada, 2008; Betancourt, Inostroza y Laborda 2008).

Como EC se utilizó un tono único de $2.8 \mathrm{kHz}$ y $80 \mathrm{~dB}(\mathrm{C})$ utilizado para las cuatro cajas durante la experimentación.

\section{Procedimiento}

\begin{tabular}{|c|c|c|c|c|c|c|}
\hline Grupos & LB1-LB2 & $\begin{array}{c}\text { Fase } \\
\text { Pavloviana }\end{array}$ & $\begin{array}{c}\text { Test } \\
\text { Medición } \\
\text { Asociación }\end{array}$ & \multicolumn{2}{|c|}{$\begin{array}{c}\text { Fase } \\
\text { Operante }\end{array}$} & \multicolumn{2}{|c|}{$\begin{array}{c}\text { Test de } \\
\text { Transferencia }\end{array}$} \\
\hline EXP & $\begin{array}{c}\text { Sin Etanol } \\
\text { Con Etanol }\end{array}$ & EC-EI & EC & $\begin{array}{c}\text { Palanca- } \\
\text { Refuerzo } \\
\text { (RF-5) }\end{array}$ & $\begin{array}{c}2 \text { min } \\
\text { sin } \\
\text { EC }\end{array}$ & $\begin{array}{c}2 \text { min } \\
\text { con } \\
\text { EC }\end{array}$ \\
\hline CON & $\begin{array}{c}\text { Sin Etanol } \\
\text { Con Etanol }\end{array}$ & $\begin{array}{c}\text { Aleatorio } \\
\text { EC/EI }\end{array}$ & EC & $\begin{array}{c}\text { Palanca- } \\
\text { Refuerzo } \\
\text { (RF-5) }\end{array}$ & $\begin{array}{c}2 \text { min } \\
\text { sin } \\
\text { EC }\end{array}$ & $\begin{array}{c}2 \text { min } \\
\text { EC } \\
\text { EC }\end{array}$ \\
\hline
\end{tabular}

Tabla 1: Diseño Experimental: EXP: Grupo experimental; CON: Grupo Control; EC: Estímulo Condicionado (Tono); EI: Estimulo Incondicionado; RF-5: Programa de Reforzamiento Razón fija 5 . 


\section{Fase Pavloviana}

Línea Base: Previo a la fase de adquisición de asociación pavloviana ("tolerancia”) se estableció una línea base 1 (LB1) y una línea base 2 (LB2) tanto para los sujetos del grupo experimental como para los del grupo control, la cual consistió en medir el grado de deslizamiento de cada sujeto en el plano de deslizamiento sin el EI (LB1) y con el EI (LB2) respectivamente en tres ensayos con un intervalo de dos minutos entre cada medición.

Adquisición de la asociación pavloviana: Esta fase se desarrolló durante 21 días, en los cuales se realizó una sesión diaria. En el grupo experimental cada sesión comenzó con la inyección de la dosis de etanol. Pasado un minuto de la inyección se puso al sujeto en el plano de deslizamiento por cinco minutos, tiempo durante el cual fue presentado el tono (EC). El grupo control recibió el mismo tratamiento utilizado con el experimental, con la diferencia de que la mitad de las veces se les inyectó la solución de etanol, mientras que la otra mitad se les inyectó suero fisiológico, con lo cual se consiguen presentaciones aleatorias del EI que permiten controlar la asociación.

Test: Medición de asociación ("tolerancia”). Finalizada esta fase, ambos grupos fueron inyectados con la dosis de etanol. Pasado un minuto de la inyección se puso al sujeto en el plano de deslizamiento y se realizaron las mediciones en los minutos 2, 4 y 6.

\section{Fase Operante}

En esta fase se moldeó la operante de apretar palanca. Se realizaron tres sesiones, hasta establecer un programa RF5.

\section{Test de transferencia}

La influencia del EC sobre la conducta operante fue medida en esta fase. Esta sesión duró cuatro minutos durante los cuales los sujetos de ambos grupos fueron introducidos a la caja operante y se les permitió presionar palanca libremente en ausencia del EC. Luego de transcurridos dos minutos, el EC fue presentado sin cesar hasta pasados dos minutos. Se midió la tasa de respuesta durante los dos primeros minutos tanto de ausencia del EC como durante su presentación. 


\section{Resultados}

\section{Fase Pavloviana}

Los resultados de esta fase están ilustrados en la Fig. 1 en la cual se muestran la LB1 y LB2 (antes y durante el EI en la primera sesión), además del test realizado durante la última sesión. Se aplicó la prueba $\mathrm{T}$ para muestras relacionadas para las líneas base y el test entre los distintos grupos. Se encontraron diferencias significativas entre la LB1 y LB2 tanto del grupo experimental $t(8)=14.78, \mathrm{p}<0.00$ como del control $t(8)=7.75, \mathrm{p}<0.01$ así como también entre la LB2 y el test del grupo experimental $t(8)=-5.61$, $\mathrm{p}<0.01$. En cambio no se encontraron diferencias significativas entre la LB2 y el test en el grupo control. Estos resultados indican que el grupo experimental logró una asociación pavloviana entre el tono (EC) y el efecto de la droga en el organismo (EI), a diferencia del grupo control. Adicionalmente es importante aclarar que se eliminó la primera de las tres mediciones de las líneas base y el test debido a que un ANOVA de medidas repetidas sobre las tres mediciones de la línea base con etanol para ambos grupos indica diferencias significativas $F(2,44)=8.2788, p=.00089$. Comparaciones entre la primera, la segunda y tercera medición juntas indican una diferencia significativa $F(2,44)=15.27, \mathrm{p}=.00075$. La comparación entre la segunda y la tercera medición no muestra diferencias estadísticamente significativas.

\section{Fase Pavloviana}

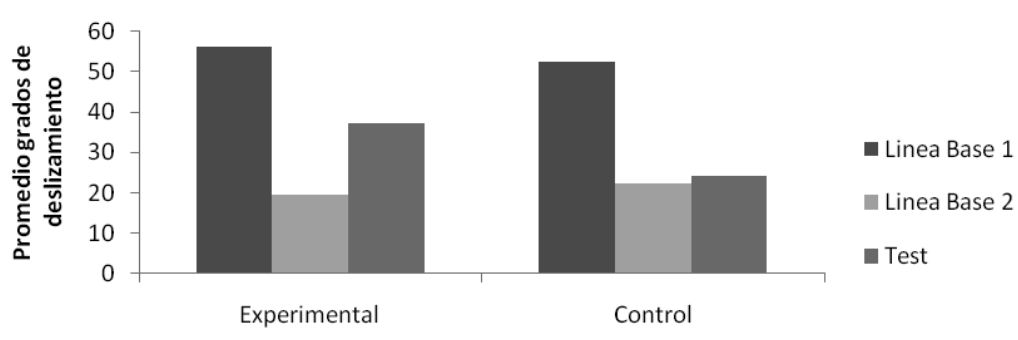

Figura. 1. Promedio de grados de deslizamiento en ambos grupos experimental y control tomados durante $L B 1, L B 2$ y test. 


\section{Test de transferencia}

En la Figura 2 se muestra la tasa de respuesta de los sujetos durante el test de transferencia. En ésta se ilustra el promedio del número de respuesta de todos los sujetos durante los dos primeros minutos (previo a presentación del EC) y los dos minutos siguientes en que se exponen el EC, tanto del grupo experimental como el de control. Se aplicó una prueba $\mathrm{T}$ para muestras relacionadas, encontrándose diferencias significativas en el grupo experimental entre la tasa de respuesta previa a la presentación del EC y durante la presentación del EC $t(8)=-2,23, \mathrm{p}=.04$. No se encontraron diferencias significativas para el grupo control. El análisis estadístico indica que la presentación del EC, cuando fue asociado previamente al efecto del etanol (EI), aumenta la conducta operante, como es el caso del grupo experimental. En cuanto al grupo control, no produce cambio en la tasa de respuesta.

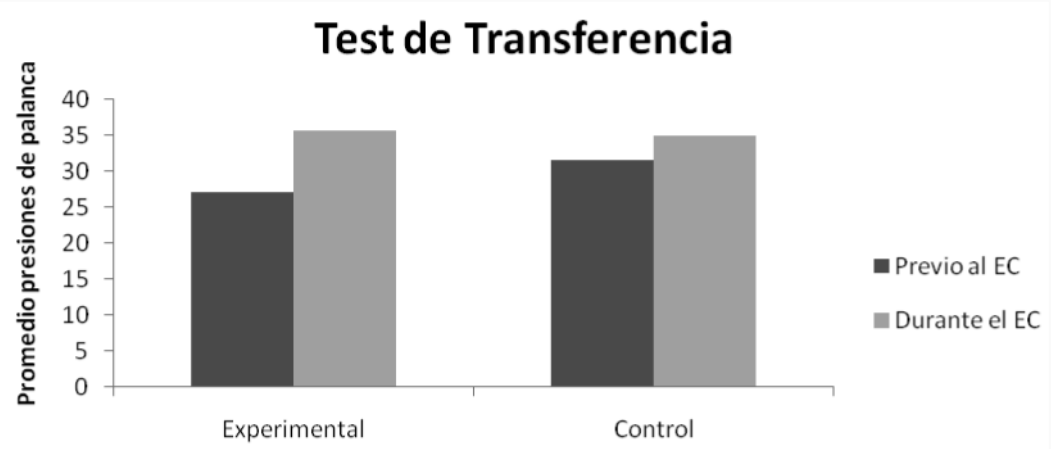

Figura 2. Promedio de presiones de palanca previo a la presentación del EC y durante la presentación del EC para ambos grupos.

\section{Discusión}

Los resultados de la presente investigación permiten sostener que los sujetos del grupo experimental, quienes tuvieron presentaciones contingentes del EC y del EI, desarrollaron un aprendizaje asociativo de respuestas compensatorias de tolerancia a los efectos atáxicos del etanol frente al estímulo condicionado tono, a diferencia de los sujetos del grupo control, quienes, al tener presentaciones aleatorias del EC y el EI no desarrollaron respuestas compensatorias frente al $\mathrm{EC}$, lo que se evidencia en que no existen diferencias significativas entre la LB2 y el test de tolerancia (ver Figura 1). 
Por otro lado, se observa que en el grupo experimental la exposición al estímulo condicionado contiguo a la emisión de la respuesta operante aumentó la tasa de ésta en comparación con la tasa de respuesta en ausencia del EC, a diferencia del grupo control, en el cual no se observan diferencias significativas en la tasa de respuesta operante en ausencia del EC y frente al EC, lo que es consistente con las predicciones de la moderna teoría de los dos procesos (Rescorla \& Solomon, 1967) puesto que no hubo asociación entre el EC y el EI en la fase pavloviana.

Estos resultados aportan evidencia experimental de que las claves (ECs) presentes al momento del consumo se asocian con los efectos del etanol en el organismo, lo que se aprecia en el desarrollo de respuestas compensatorias al efecto atáxico del etanol en el grupo experimental. Del mismo modo aportan evidencia de que eventos asociados clásicamente, si éstos son presentados contiguos a la emisión de una operante, modifican su tasa, en este caso aumentándola. Esto es consistente con los resultados encontrados por Corbit y Janak (2007), quienes observan un aumento de la respuesta operante para eventos apetitivos naturales (por ejemplo, sucrosa) en presencia de ECs asociados al etanol. También son concordantes con los resultados de Krank (2003), que observa un aumento de la respuesta de consumo de etanol frente a ECs asociados a éste y con los hallazgos de Hellemans, Dickinson y Everitt (2006) quienes observan un aumento de la búsqueda de droga frente a estímulos asociados a la heroína.

Como se mencionó en el párrafo precedente, estos resultados son consistentes con las predicciones de la moderna teoría de los dos procesos de Rescorla y Solomon (1967), la que postula que el condicionamiento clásico provee de un Estado Emocional Central (EEC) que depende del tipo de EI que se haya utilizado y su relación de contingencia con los EC. Además postula que el condicionamiento pavloviano provee de las bases motivacionales al condicionamiento operante, lo cual se produce en cada ensayo de condicionamiento operante en el que se asocian claves presentes durante la entrega de las consecuencias (positivas /negativas). Por lo tanto, el estado motivacional va a depender del tipo de consecuencia y su relación de contingencia con la respuesta. En esta línea de razonamiento, entonces si se tiene un estado motivacional particular en una asociación operante, 
al presentar un evento condicionado clásicamente (un EC), éste sumaría o restaría el efecto sobre la operante, vale decir, el Estado Emocional Central gatillado por el EC influye o afecta la tasa de emisión de la respuesta operante que se esté ejecutando. Desde este modelo, y frente a los resultados de la presente investigación, los ECs asociados al efecto del etanol elicitarían un estado emocional central placentero de contingencia positiva, lo cual, al ser agregado al estado motivacional en la ejecución de una operante mantenida por un programa de reforzamiento positivo, daría como resultado el aumento de la respuesta operante, lo que de hecho ocurrió en esta investigación. Esto es consistente con las predicciones hechas por las teorías del incentivo en que el consumo de droga está más bien relacionado con las propiedades apetitivas de ésta (Hellemans, Dickinson \& Everitt, 2006).

Otra variante importante a considerar en cuanto al valor hedónico de las drogas es que, al igual que cualquier otro proceso de aprendizaje operante, se asocian claves a los eventos, las cuales pasan a tener propiedades placenteras o displacenteras y al ser contingentes a la emisión de una conducta, se alteraría la probabilidad de emisión de ésta a futuro. En este caso, situaciones o eventos asociados a la droga o carencia de ésta alterarían la respuesta de búsqueda o consumo de las drogas. De este modo se demuestra que las claves presentes en el momento del consumo de etanol se asocian clásicamente, por lo que pasarían a ser reforzadores condicionados.

Por otro lado es importante tener en cuenta que las propiedades motivacionales que adquiere un EC están relacionadas con el tipo de droga que se utilice y su relación de contingencia, por lo tanto las diversas preparaciones experimentales y los resultados esperados en próximas investigaciones deben tener en cuenta este punto. $\mathrm{Al}$ respecto, Baldwin y Koob (1993) presentan resultados consistentes con la moderna teoría de los dos procesos, pues encontraron que ratas opiáceo-dependientes disminuyen su respuesta para la comida cuando ésta es presentada con estímulos pareados con abstinencia a la droga. Es decir, una relación de contingencia negativa con un evento apetitivo, lo cual genera un estado motivacional hedónico negativo, el que se resta al estado motivacional hedónico positivo producto de una contingencia apetitiva positiva. Si bien estos resultados 
difieren de los encontrados en la presente investigación, esta diferencia puede ser entendida a partir de que distintas drogas (por ejemplo, etanol versus opiáceos) pueden asociarse de distinta manera (por ejemplo, aversivamente versus apetitivamente) con los estímulos.

\section{Referencias bibliográficas}

Aнmed, S. \& Коов, G. (1997). Cocaine- but not food-seeking behavior is reinstated by stress after extinction. Psychopharmacology, 132, 289-295.

Baldwin, H.A. y Коов, G.F. (1993). Rapid induction of conditioned opiate withdrawal in the rat. Neuropsychopharmacology, 8, 15-21.

Bechara, A., Nader, K. \& Van Der Kooy, D. (1998). A two-separatemotivational-systems hypothesis of opioid addiction. Pharmacology Biochemistry and Behavior, 59, 1-17.

Betancourt, R. (2006). Condicionamiento clásico y drogas: Modulación de los procedimientos de extinción a la tolerancia y síntomas de abstinencia al etanol en ratas. En C. Decouvieres. (Ed.): Tesis doctorales en psicología. Santiago de Chile: Praxis.

Betancourt, R. Corada, L., Dominichetti, J., Laborda, M., Martínez, G. y Miguez, G. (2008). Efecto de la extinción en múltiples contextos sobre la renovación de la tolerancia asociativa al etanol. Psicothema, 20, 285-289.

Betancourt, R., Díaz, C.G., Quezada, V. (2008). Claves interoceptivas y exteroceptivas en la tolerancia al efecto atáxico del etanol en ratas. Psicothema, 20, 807-811.

Betancourt, R., Inostroza, M. y Laborda, M. (2008). Modulación contextual de la tolerancia asociativa al etanol. Revista Latinoamericana de Psicología, 40, 243-257.

Childress, A.R., Mozley, P.D., McElgin, W., Fitzgerald, J., Reivich, M. \& O'Brien, C.P. (1999). Limbic activation during cue-induced cocaine craving. American Journal of Psychiatry. 156, 11-18.

Corbit, L. \& Janak, L. (2007). Ethanol-associated cues produce general pavlovian-instrumental transfer. Alcoholism: Clinical and Experimental Research, 31, 1-9.

Crowell, C. R., Hinson, R. E. \& Siegel, S. (1981). The role of condicional drug response in tolerance to the hypothermic effect of etanol. Psychopharmacology, 73, 51-54. 
Di Ciano, P. \& Everitt, B. (2003). Differential control over drugseeking behavior by drug-associated conditioned reinforcers and discriminative stimuli predictive of drug availability. Behavioral Neuroscience, 117, 952-960.

Dickinson, A., Wood, N. \& Smith, J. (2002). Alcohol seeking by rats: action or habit? The Quarterly Journal of Experimental Psychology. 55B, 331-348.

GaWin, F.H. \& Kleber, H.D. (1986). Abstinence symptomatology and psychiatric diagnosis in cocaine abusers. Archives of General Psychiatry, 43, 107-113.

Glasner, S., Overmier, B. \& Balleine, B. (2005). The role of pavlovian cues in alcohol seeking in dependent and nondependent rats. $J$. Stu. Alcohol, 66, 53-61.

Goodison, T. y Siegel, S. (1995). Tolerance to Naloxone-Induced Suppression of Intake Learning and Cross-Tolerance to Cholecystokinin in Rats. Behavioral Neuroscience, 109, 455-465.

Hellemans, K., Dickinson, A. \& Everitt, B. (2006). Motivational control of heroin seeking by conditioned stimuli associated with withdrawal and heroin taking by rats. Behavioral Neuroscience, 1, 103-114.

Krank, M. (2003). Pavlovian conditioning with ethanol: signtracking (autoshaping), conditions incentive, and ethanol selfadministration. Alcoholism: Clinical and Experimental Research, 27, 1592-8.

Larson, S. \& Siegel, Sh. (1998). Learning and Tolerance to the Ataxic Effect of Ethanol. Pharmacology Biochemistry and Behavior, 61, 131-142.

McDonald, R. \& Siegel, S. (2004) Intra-Administration Associations and Withdrawal Symptoms: Morphine-Elicited Morphine Withdrawal. Experimental and Clinical Psychopharmacology. 12, 3-11.

MacRae, J. R. \& Siegel, S. (1997). The role of self-administration in morphine withdrawal in rats. Psychobiology, 25, 77-82.

Mayorga, P., Albañil, N. y Cómbita, L. (2006). Elección y sustitución de estímulos entre el alcohol y el alimento en ratas: una explicación del consumo de drogas desde el contexto de la economía conductual. Suma Psicológica, 13, 33-50.

Michael, J. (1963). Laboratory Studies in Operant Behavior. New York: McGraw-Hill Book Company, Inc.

Miles, F., Everitt, B., Dalley, J. y Dickinson, A. (2004). Conditioned activity and instrumental reinforcement following long-term oral consumption of cocaine by rats. Behavioral Neuroscience, 118, 1331-1339. 
Parkinson, J.A., Olmstead, M.C., Burns, L.H., Robbins, T.W. y Everitt, B.J. (I999). Dissociation in effects of lesions of the nucleus accumbens core and shell on appetitive Pavlovian approach behavior and the potentiation of condictioned reinforcement and locomotor activity by $d$-amphetamine. Journal of Neuroscience, 19 , 2401-2411.

Ramos, B., Siegel, S. \& Bueno, J. L. (2002). Occasion Setting and Drug Tolerance. Integrative Physiological y Behavioral Science, 37, 165-177.

Rescorla, R.A. \& Solomon, R.L. (1967). Two-process learning theory: Relationships between Pavlovian conditioning and instrumental learning. Psychological Review, 74, 151-182.

Robinson, T.E. \& Berridge, K.C. (2002). Addiction. Annual Review of Psychology, 14, 14.

Shaham, Y. \& MiczeK, K. (2003). Reinstatement-toward a model of relapse. Psychopharmacology, 168, 1-2.

Shalev, U., Highfield., Yap, J. \& Shaham, Y. (2000). Stress and relapse to drug seeking in rats: studies on generality of the effect. Psychopharmacology, 150, 337-346.

SIEGEL, S. (1977). Morphine tolerance acquisition as an associative process. Journal of Experimental Psychology: Animal Behavior Processes, 3, $1-13$.

Siegel, S. (1978). Tolerance to the Hiperthermic Effect of Morphine in the rat is a Learned Response. Journal of Comparative an Pshysiological Psychology, 92, 1137-1149.

Siegel, S. (1991). Feedforward processes in drug tolerance. Perspectives in Cognitive Neuroscience, 405-416. New York: Oxford University Press.

Siegel, S. (1999). Drug anticipation and drug addiction: The $1998 \mathrm{H}$. David Archibald lecture. Addiction, 94, 1113-1124.

Siegel, S. (2001). Pavlovian conditioning and drug overdose: When tolerance fails. Addiction Research and Theory, 9, 503-513.

Siegel, S. \& Larson, S. (1996). Disruption of Tolerance to the Ataxic Efftect of Ethanol by a novel Stimulus. Pharmacology, Biochemistry and Behavior, 55, 125-130.

Siegel, S. \& Ramos, B. M. (2002). Applying Laboratory Research. Drug Anticipation and the Treatment of Drug Addiction. Experimental and Clinical Psychopharmacology, 10, 162-183.

Fecha de Recepción de artículo: 25 de agosto de 2009.

Fecha de Aceptación de artículo: 09 de octubre de 2009. 
